CHERCHER, REPÉRER, AVANCER

\title{
LE GENRE PROGRAMMATIQUE
}

Sociologie de la production des programmes partisans : l'exemple de Bad Godesberg Karim Fertikh

Presses de Sciences Po (P.F.N.S.P.) | « Revue française de science politique »

2014/5 Vol. 64 | pages 905 à 928

ISSN 0035-2950

ISBN 9782724633726

Article disponible en ligne à l'adresse :

http://www.cairn.info/revue-francaise-de-science-politique-2014-5-page-905.htm

\section{Pour citer cet article :}

Karim Fertikh, « Le genre programmatique. Sociologie de la production des programmes partisans : l'exemple de Bad Godesberg », Revue française de science politique 2014/5 (Vol. 64), p. 905-928.

DOI 10.3917/rfsp.645.0905

Distribution électronique Cairn.info pour Presses de Sciences Po (P.F.N.S.P.).

(C) Presses de Sciences Po (P.F.N.S.P.). Tous droits réservés pour tous pays.

La reproduction ou représentation de cet article, notamment par photocopie, n'est autorisée que dans les limites des conditions générales d'utilisation du site ou, le cas échéant, des conditions générales de la licence souscrite par votre établissement. Toute autre reproduction ou représentation, en tout ou partie, sous quelque forme et de quelque manière que ce soit, est interdite sauf accord préalable et écrit de l'éditeur, en dehors des cas prévus par la législation en vigueur en France. Il est précisé que son stockage dans une base de données est également interdit. 


\section{LE GENRE}

\section{PROGRAMMATIQUE}

\section{SOCIOLOGIE DE LA PRODUCTION DES PROGRAMMES PARTISANS :} L'EXEMPLE DE BAD GODESBERG

\section{Karim Fertikh}

E n s'appuyant sur des recherches effectuées sur le programme de Bad Godesberg du Parti social-démocrate allemand (SPD) adopté en 1959, cet article veut proposer une démarche d'analyse des programmes politiques. Dans une perspective écologique ${ }^{1}$ et socio-historique, notre démarche entend encastrer ce type de texte dans le faisceau des relations sociales, structurées par l'histoire, qui le fait exister. Elle cherche à mettre en évidence la structuration historique des interactions politiques, c'est-à-dire à considérer qu'au-delà des événements singuliers que constitue chaque nouveau programme partisan, se laissent aussi lire les appropriations particulières de savoir-faire sédimentés, les programmes étant une forme historique du politique autant structurante que lente à s'user.

Cette contribution a d'abord une ambition proprement sociologique, inscrite dans la lignée de travaux qui appellent à dépasser l'opposition entre lectures internalistes et externalistes des textes ${ }^{2}$. On ne croit plus, à l'instar d'un Jean Thibaudet, que «la politique, ce sont des idées » et que les partis, «expressions de grandes familles d'idées politiques» ont un programme qu'ils réalisent avant de "s'enliser $»^{3}$. Une analyse sociologique des programmes inscrit notamment les idées politiques dans une matérialité saisissable et analysable dans sa production, sa diffusion et ses usages. L'histoire sociale des idées politiques a donné lieu en France, ces dernières années, à un ensemble de travaux rénovant les instruments mobilisés pour comprendre la dynamique de la production d'idées politiques, essentiellement à partir de l'analyse de la construction de «courants» ou de la circulation et de l'usage de biens symboliques ${ }^{4}$. Les programmes constituent alors un observatoire pour une telle sociologie des idées politiques, pour peu qu'on veuille y voir non pas seulement des textes à interpréter

\footnotetext{
1. Frédéric Sawicki, Les réseaux du parti socialiste. Sociologie d'un milieu partisan, Paris, Belin, 1997, p. 13.

2. Pierre Bourdieu, Les règles de l'art. Genèse et structure du champ littéraire, Paris, Seuil, 1992, p. 318-319 ; Patrick Lehingue, Bernard Pudal, "Retour(s) à l'expéditeur. Éléments d'analyse pour la déconstruction d'un "coup" : la "Lettre à tous les Français" de François Mitterrand", dans CURAPP, Communication politique, Paris, PUF, 1991, p. 163-182.

3. Cité dans Jean Charlot, Les partis politiques, Paris, Armand Colin, 1971, p. 58-60.

4. Les ambitions de ce programme de recherche en histoire sociale des idées politiques ont été formulées dans deux textes: Frédérique Matonti, «Plaidoyer pour une histoire sociale des idées politiques», Revue d'histoire moderne et contemporaine, 59 (4 bis), 2012, p. 85-104; et Bernard Pudal, " De I'histoire des idées politiques à I'histoire sociale des idées politiques", dans Antonin Cohen, Bernard Lacroix, Philippe Riutort (dir.), Les formes de l'activité politique. Éléments d'analyse sociologique. XVIIle-xxe siècle, Paris, PUF, 2006, p. 185-192. L'ambition d'une rénovation de l'histoire des idées, en particulier d'une meilleure articulation avec l'histoire sociale fait également l'objet de réflexions en Allemagne. Cf. pour un état des lieux : Lutz Raphael, «"Ideen als gesellschaftliche Gestaltungskraft im Europa der Neuzeit". Bemerkungen zur Bilanz eines DFG-Schwerpunktprogramms ", dans Lutz Raphael, Heinz-Elmar Tenorth (Hrsg.), Ideen als gesellschaftliche Gestaltungskraft im Europa der Neuzeit. Beiträge für eine erneuerte Geistesgeschichte, Munich, Oldenbourg, 2006, p. 11-30.
} 
mais le produit de stratégies d'acteurs situés dans des espaces organisationnels, politiques (ceux de la compétition électorale ou ceux des luttes sociales ${ }^{1}$ ) et intellectuels ${ }^{2}$. Ils sont aussi le support de relations sociales engageant des acteurs qui se servent du texte dans leurs interactions : au sein d'un parti politique lors de sa production (experts scientifiques, services de propagande ou de communication ${ }^{3}$ ) ou lors de sa diffusion (militants, dirigeants, opposants, journalistes, historiens, etc.).

Notre recherche propose un détour socio-historique afin de dénaturaliser l'évidence du programme et d'envisager correctement sa spécificité et sa structuration historiques ${ }^{4}$. Dans de nombreux pays européens, le $19^{\mathrm{e}}$ siècle est celui de l'invention et de l'apprentissage de la démocratie. En France, des travaux socio-historiques ont déjà été consacrés au vote, à la civilisation électorale de la compétition politique ${ }^{5}$ ou à la construction du lien entre vote et opinion $^{6}$. L'historiographie a largement exploré les questions de politisation, au sens d'une fabrication du citoyen électeur ou d'acclimation de la compétition politique au niveau local ${ }^{7}$, ou encore celle des technologies propres au vote dans sa conception moderne ${ }^{8}$. La professionnalisation du métier politique et l'invention de l'homme politique moderne ont également fait l'objet d'un investissement récent des sciences politique et historique ${ }^{9}$. En revanche, la question du programme reste, elle, relativement peu analysée, sinon en creux par des travaux qui notent son faible usage dans les contextes électoraux pré-modernes (puisque, «en temps ordinaires, les électeurs ne votent pas pour une politique ${ }^{10}$ ) ou modernes ${ }^{11}$,

1. Pierre Bréchon, «Partis politiques et idéologies», dans Pierre Bréchon (dir.), Le discours politique en France. Évolution des idées partisanes, Paris, La Documentation française, 1994, p. 5-15, ici p. 7.

2. On renvoie notamment à John G. A. Pocock, Political Thought and History. Essays on Theory and Method, Cambridge, Cambridge University Press, 2009.

3. Sur l'importance de la division du travail dans le fonctionnement politique, cf. entre autres: Guillaume Sacriste, La république des constitutionnalistes, Paris, Presses de Sciences Po, 2011 ; Jean-Baptiste Legavre, "La quête des origines. Retour sur la fondation de la communication politique experte en France ", Questions de communication, 7, 2005, p. 323-344; Arnaud Mercier, "La communication politique en France. Un champ de recherche qui doit encore s'imposer", L'Année sociologique, 51, 2001, p. 355-363.

4. Yves Déloye, "À la recherche de la temporalité perdue", EspacesTemps, 76-77, 2001, p. 16-27.

5. Olivier Ihl, "L'urne et le fusil. Sur les violences électorales lors du scrutin du 23 avril 1848 », Revue française de science politique, 60 (1), février 2010, p. 9-35.

6. Cf. en particulier: Yves Déloye, «Pour une sociologie historique de la compétence à opiner "politiquement". Quelques hypothèses de travail à partir de l'histoire électorale française ", Revue française de science politique, 57 (6), décembre 2007, p. 775-798.

7. Sans prétendre à l'exhaustivité, cf. par exemple : Maurice Agulhon, La République au village, Paris, Seuil, 1979

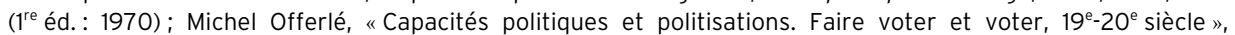
Genèses, 67, 2007, p. 131-149, et 68, 2007, p. 145-160; Patrice Gueniffey, Le nombre et la raison. La Révolution française et les élections, Paris, Éditions de I'EHESS, 1993; Christine Guionnet, L'apprentissage de la politique moderne. Les élections municipales sous la monarchie de Juillet, Paris, L'Harmattan, 1997; Laurent Le Gall, "L'élection au village dans la France du xıxe siècle. Réflexions à partir du cas finistérien ", Revue d'histoire du XIXe siècle, 43, 2011, p.17-39; Paula Cossart, Le meeting politique. De la délibération à la manifestation (1868-1939), Rennes, Presses Universitaires de Rennes, 2010.

8. Malcolm Crook, Tom Crook, "L'isoloir universel ? La globalisation du scrutin secret au xixe siècle ", Revue d'histoire du Xixe siècle, 43, 2011, p. 41-55.

9. Éric Phélippeau, L'invention de la politique moderne. Mackau, l'Orne et la République, Paris, Belin, 2002; Michel Offerlé (dir.), La profession politique. XIx -xx siècles, Paris, Belin, 1999.

10. Paul Veyne, Le pain et le cirque. Sociologie historique d'un pluralisme politique, Paris, Seuil, 1976, p. 399; Karl Wilhelm Weeber, La fièvre électorale à Pompéi, Paris, Les Belles Lettres, 2011.

11. Jean Vigreux, "Les campagnes françaises et la politiques (1830-1914)", Parlement(s). Revue d'histoire politique, 5, 2006, p. 54-72, ici p. 70. On renvoie aux analyses de Christine Guionnet sur l'incompatibilité du pluralisme et de la compétition électorale avec une conception holiste de la société : «Élections et apprentissage de la politique : élections municipales sous la monarchie de Juillet », Revue française de science politique, 46 (4), août 1996, p. 555-579, en particulier p. 571; Philippe Secondy, « Royalisme et innovations partisanes. Les "blancs 
Claire Andrieu en signalant la rareté avant l'avènement de la Troisième République ${ }^{1}$. Peutêtre sous l'effet de l'idée de Sonderweg, le courant socio-historique apparaît moins développé pour l'analyse de l'Allemagne $e^{2}$. La seconde moitié du $19^{\mathrm{e}}$ siècle y est aussi, à des rythmes divers suivant ses États, celle de l'apprentissage du vote et des formes modernes de courtage électoral. Les historiens ont montré que la social-démocratie, produit de la Révolution de 1848, y constitue un vecteur central de cet apprentissage démocratique ${ }^{3}$. Il est donc intéressant de prendre, en l'inscrivant sur une longue période, la social-démocratie allemande comme terrain d'analyse de la production et de l'usage des programmes.

Faire une socio-histoire du programme comme instrument "pratique et cognitif de l'élection ${ }^{4}$ revient ainsi à contribuer à l'analyse de l'émergence du répertoire d'actions démocratiques et au mouvement d'innovations qui accompagne le développement de la démocratie représentative. Cette perspective invite dès lors à déplacer le regard porté habituellement sur les programmes : au-delà des mots et des prises de position, ils peuvent être saisis sous l'angle des procédures qui amènent à la production d'un texte et à sa consécration comme programme. Notre recherche considère le programme comme une transaction avec une organisation et son passé objectivé dans des acteurs, des routines, un espace de références textuelles, avec des normes régulatrices du texte, contraignant les formes d'écriture et de lecture ${ }^{5}$, ou encore avec, au-delà des électeurs, des " pairs » ou des catégories d'acteurs signifiantes pour les rédacteurs des programmes.

Notre contribution veut mettre en évidence les appropriations du genre programmatique par des acteurs qui le transforment au fil du temps. Cette démarche fait écho à des travaux d'historiens qui poursuivent l'ambition d'analyser la fabrication des textes programmatiques. Dans ces travaux, la description fine des contextes de production des textes repose sur la minutie du travail archivistique. L'histoire du programme du Conseil national de la Résistance (CNR) de Claire Andrieu, qui suit les interactions productrices du texte, en est sans doute l'exemple le plus précis ${ }^{6}$, même si l'on trouverait un effet identique dans l'ensemble des contributions de l'ouvrage dirigé par Alain Bergounioux et Danielle Tartakowsky consacré à l'union de la gauche et au programme commun de $1972^{7}$. Ces travaux partagent tous deux l'intérêt d'enraciner les textes qu'ils analysent dans la culture politique et organisationnelle de leur époque. Ils étudient aussi bien les stratégies concrètes des acteurs, les relations entre les négociateurs et leur organisation ou encore les réceptions de ces textes. Portant sur ces programmes comme événements singuliers, ces travaux ne proposent pas encore de modèle systématique d'analyse de ce type de texte politique.

du Midi” à la fin du $19^{e}$ siècle ", Revue française de science politique, 53 (1), février 2003, p. 73-99, en particulier p. 88 et suiv.

1. Claire Andrieu, Le programme commun de la Résistance. Des idées dans la guerre, Paris, Les Éditions de l'érudit, 1984, p. 9.

2. On citera : Larry Jones, James Retallack (eds), Elections, Mass Politics and Social Changes in Modern Germany. New Perspectives, Cambridge, Cambridge University Press, 1992 ; Margaret Malvina Anderson, Praticing Democracy. Elections and Political Culture in Imperial Germany, Princeton, Princeton University Press, 2000.

3. Thomas Welskopp, Das Banner der Brüderlichkeit. Die deutsche Sozialdemokratie vom Vormärz bis zum Sozialistengesetz, Bonn, Dietz, 2000 ; Bernd Warneken, "Massentritt. Zur Körpersprache von Demonstranten in Kaiserreich », dans Peter Assion (Hrsg.), Transformationen der Arbeiterkultur, Marbourg, Jonas Verlag, 1988, p. 64-80.

4. Christophe Voilliot, "Introduction", Revue d'histoire du xixe siècle, 43, 2011, p. 7-13, dont p. 11.

5. Wolgang Iser, Der implizite Leser, Stuttgart, Suhrkamp, 1979.

6. C. Andrieu, Le programme commun..., op. cit.

7. Alain Bergounioux, Danielle Tartakowsky (dir.), L'union sans unité. Le programme commun de la gauche 1963-1978, Rennes, Presses Universitaires de Rennes, 2012. 
En revanche, cet article voudrait esquisser une piste de recherche alternative aux travaux de la science politique internationale incarnée par le Comparative Manifesto Project (CMP), structurés d'abord autour d'Ian Budge ${ }^{1}$ et étalon de la recherche en la matière. Les programmes ont, bien sûr, suscité l'intérêt de la recherche en science politique avant la mise en place de ce réseau, notamment dans les analyses du «langage du pouvoir $»^{2}$ et des travaux ont préfiguré l'entreprise systématique du CMP en travaillant l'objet "programme » par la statistique textuelle ${ }^{3}$. Par rapport aux recherches du CMP, notre démarche se distingue en renonçant à s'inscrire dans le cadre d'une théorie du choix rationnel appliquée à la démocratie $^{4}$ assimilant le programme à une offre politique destinée à permettre l'arbitrage des électeurs. Les programmes sont alors vus comme l'expression de préférences, et l'on ignore la manière dont ils sont produits ${ }^{5}$. En outre, le CMP s'intéresse aux programmes comme indicateurs du positionnement des partis. En découle une dématérialisation de ce qui est considéré comme "manifesto». En cherchant à combler les lacunes du corpus de programmes officiels, les pratiques de recherche du CMP codent, en effet, les déclarations de dirigeants, les brouillons des programmes, les publicités dans les journaux voire le contenu des tracts, des programmes régionaux ou des résumés journalistiques des programmes ${ }^{6}$. Enfin, dans notre optique, le sens d'un programme ne peut être fixé une fois pour toute, et codé de manière « objective ». La signification d'un texte émerge du " processus de lecture " ${ }^{7}$, c'est-à-dire est l'un des effets de l'appropriation par des acteurs concrets d'un texte empirique ${ }^{8}$.

Au-delà de la pétition de principe méthodologique que notre positionnement exprime, le projet d'une recherche contextualisée sur les programmes se fonde sur une analyse réalisée sur le programme de Bad Godesberg, non pas comme événement en "rupture » avec la tradition social-démocrate, mais comme texte inscrit dans l'histoire longue des programmes sociaux-démocrates. Pour ce faire, nous nous appuyons notamment sur une recherche réalisée sur les programmes des années 1920 (programme de Görlitz en 1921 et programme d'Heidelberg en 1925) à partir des archives Bernstein (Institut international d'histoire sociale à Amsterdam, IISG, série N). Pour l'étude de Bad Godesberg, nous avons mobilisé des fonds archivistiques importants réunis dans diverses institutions (entre autres, les archives de la démocratie sociale à Bonn, AdsD, et l'IISG) ${ }^{9}$. Nous avons également eu

1. Cf. Ken Newton, «lan Budge. A Life of Writing and Organizing, Walking, Talking", dans Judith Bara, Albert Weale (eds), Democratic Politics and Party Competition. Essays in honour of lan Budge, Abingdon, Routledge/ ECPR, 2009, p. 21-32.

2. Harold Lasswell, "Langage of Power ", dans Harold Lasswell, Nathan Leites (eds), Langage of Politics. Studies in Quantitative Semantics, Cambridge, The MIT Press, 1965, p. 3-20 ; Serge Tchakhotine, Le viol des foules par la propagande politique, Paris, Gallimard, 1939.

3. Antoine Prost, Vocabulaire des proclamations électorales de 1881, 1885 et 1889, Paris, PUF, 1974 ; Kenneth Janda, Political Parties. A Cross-National Survey, New York, The Free Press, 1980.

4. Cf. la mise au point de Judith Bara, "Do Parties Reflect Public Concerns?», dans J. Bara, A. Weale (eds), Democratics Politics..., op. cit., p. 105-121, en particulier p. 105-106.

5. Nicolas Merz, Sven Regel, «Die Programmatik der Parteien», dans Oskar Niedermayer (Hrsg.), Handbuch Parteienforschung, Wiesbaden, Springer, 2013, p. 211-238.

6. Kostas Gemenis, « What to Do (and Not to Do) with the Comparative Manifestos Project Data », Political Studies, 61 (1), 2013, p. 3-23; Martin Hansen, «Back to the Archives? A Critique of the Danish Part of the Manifesto Dataset ", Scandinavian Political Studies. A Journal for the Nordic Political Science, 31 (2), 2008, p. 201-216.

7. Wolfgang Iser, L'appel du texte, Paris, Allia, 2012 (1'e éd. : 1969), p. 11.

8. Cf. Stanley Fish, "Is There a Text in this Class ?" The Authority of Interpretive Community, Cambridge, Harvard University Press, 1980.

9. Karim Fertikh, "Le congrès de Bad Godesberg. Contribution à une socio-histoire des programmes politiques ", thèse de doctorat en sciences sociales, Paris, EHESS, 2012. 
recours aux publications, notamment aux brochures ou aux ouvrages scientifiques des membres des commissions. Sous la direction d'Erich Ollenhauer (1901-1963, président du SPD de 1952 à sa mort $^{1}$ ), le SPD installe en 1955 une commission programmatique chargée de fournir de nouvelles lignes directrices au parti. Celle-ci soumet en 1958 la première version d'un texte, modifié l'année suivante avant d'être soumis au vote des délégués du SPD dans un congrès se déroulant à Bad Godesberg, dans la banlieue de Bonn. Cette recherche a eu pour atout le caractère bureaucratisé du parti, et la participation au travail sur le programme d'acteurs habitués à l'écrit et producteurs aussi bien de textes publiés que de lettres ou de mémoires. La disponibilité de ces archives découle de la perte d'intérêt stratégique des transactions réalisées pour produire le programme de 1959: de nombreux documents frappés du tampon «confidentiel» montrent l'enjeu que constitue le secret des tractations, jusque très tard dans la production du texte. Disposer d'archives a pour contrepartie la disparition de nombreux acteurs qui ont pris part à la production du programme, et, pour les deux acteurs que nous avons retrouvés, l'imprécision des souvenirs. Plutôt que de les interroger sur les détails des arrangements au sein de la commission programmatique, nous avons opté pour un entretien ouvert, ressemblant plus à un récit de vie et permettant d'objectiver les rapports au Parti de ces acteurs. En revanche, nous avons largement exploré les fonds privés d'acteurs impliqués dans la production du programme pour éclairer les comptes rendus officiels et l'activité bureaucratique qui entoure sa production et sa diffusion.

\section{Une chronologie}

\begin{tabular}{l|l}
\hline 1875 & $\begin{array}{l}\text { Programme de Gotha (du SAPD) } \\
1891\end{array}$ \\
1921 & Programme d'Erfurt \\
1925 & Programme de Görlitz \\
$1955-1958$ & Programme d'Heidelberg \\
1958 & Commission programmatique du SPD \\
Mai 1959-automne 1959 & Projet de programme de Stuttgart \\
$13-15$ novembre 1959 & Seconde commission programmatique du SPD \\
\hline
\end{tabular}

L'article s'articule en trois temps. Il s'intéresse, d'abord, à la codification du genre programmatique en Allemagne et à la manière dont ces contraintes formelles sont appropriées par les rédacteurs du programme de 1959. Ensuite, il met en évidence l'importance de la prise en compte de l'espace organisationnel pour comprendre la production d'un programme politique ainsi que les effets de la scientifisation du politique sur la production doctrinale. Enfin, il suit le texte "à l'air libre » en s'intéressant aux significations acquises par le programme de Bad Godesberg à travers les usages que l'on fait de lui sur différentes scènes sociales.

1. Un tableau à la fin de cet article recense les contributeurs au programme de Bad Godesberg mentionnés ici. 


\section{Un genre programmatique}

$\mathrm{N}$

ous sommes habitués à penser les programmes comme des objets familiers, des catalogues de mesures proposées par des partis lors des élections. Cette familiarité est le produit d'un lent processus de définition de cette forme partisane. Pour la science politique, rappeler que les programmes ont une histoire amène à analyser les conditions sociales sous lesquelles un document écrit acquiert les caractéristiques et le nom d'un programme politique. L'émergence des programmes comme forme politique correspond à l'invention d'un genre de texte qui se plie à des « conventions notoires » définissant l'horizon d'attente des lecteurs, et donc contraignantes pour les producteurs ${ }^{1}$.

Les moments de construction des pratiques éclairant les éléments qui les définissent ${ }^{2}$, nous nous proposons donc de revenir sur l'émergence de cet instrument de l'élection à partir du $19^{\text {e }}$ siècle $^{3}$. Nous nous appuyons sur des manuels et des recueils de programmes produits par des acteurs politiques, des partis ou des historiens à l'époque de l'institutionnalisation de l'histoire des partis politiques au tournant du siècle. Au-delà de cette question de codification et de genèses, nous cherchons à mettre en évidence la manière dont les producteurs du programme de Bad Godesberg rencontrent ces conventions du genre programmatique dans leur «matérialité »"

\section{La standardisation des programmes politiques au $19^{\mathrm{e}}$ siècle}

Un détour par les mots peut donner une idée de la nouveauté du genre programmatique. Le terme ne fait son apparition dans une signification politique que tardivement, que cela soit en France ou en Allemagne. Le mot apparaît en langue allemande sous la forme Programmata dès le $18^{\mathrm{e}}$ (dictionnaire Ziedler, 1712), et est germanisé en Programm au $19^{\mathrm{e}}$ siècle. Cependant, il n'acquiert sa signification moderne de proclamation politique que dans la seconde moitié de ce siècle. En 1824 comme en 1856, l'encyclopédie Brokhaus (Allgemeine deutsche Enzyklopädie für gebildete Stände), référence en langue allemande, en limite la signification aux feuillets volants ou affichés sur les murs qui annoncent le déroulement d'une festivité ou d'une manifestation publiques, particulièrement universitaires. Dans sa douzième édition en 1886, l'encyclopédie en donne comme sens dérivé, celui d' « exposé de principes politiques » (ce que ne fait pas encore à la même époque le dictionnaire des frères $\mathrm{Grimm}^{5}$ ).

La dénomination ne marque pas à elle seule la stabilisation de la forme. En particulier, les programmes désignent longtemps, au-delà de textes précis, des courants d'idées, dont la production n'est pas le fait d'une organisation bien délimitée : parler de programme est une manière de styliser des "opinions " émises, par exemple, dans les salons bourgeois ou dans des journaux. Ce n'est que tardivement que se précise le lien des programmes avec la compétition et les partis politiques. Ainsi, le «programme conservateur » du philologue allemand

1. Hans Robert Jauss, "Histoire de la littérature ", dans Pour une esthétique de la réception, Paris, Gallimard, 1978 , p. 22-88, ici p. 56 et suiv.

2. Sur ce point, cf. Michel Offerlé, "De l'histoire électorale à la socio-histoire des électeurs ", Romantisme, 135, 2007, p. 61-69, ici p. 66.

3. Pour une étude détaillée de la genèse des programmes en France, on renvoie à Karim Fertikh, Mathieu Hauchecorne, "Codification et genèses d'un genre programmatique. Les professions de foi des députés français de la Seine, du Nord et de la Vendée en 1881, 1907 et 1919 ", dans Karim Fertikh, Mathieu Hauchecorne, Nicolas Bué, Faire, défaire les programmes. Genèses, coproduction, usages, à paraître.

4. Roger Chartier, «Le monde comme représentation», Annales ESC, 44 (6), 1989, p. 1503-1520, ici p. 1513.

5. Jakob Grimm, Wilhelm Grimm, Deutsches Wörterbuch, Leipzig, Verlag von S. Hirzel, 1889, vol. 7. 
Paul de Lagarde n'est écrit au nom d'aucun parti ; bien au contraire, il appelle le ralliement des conservateurs autour du programme formulé, car, écrit Paul de Lagarde " chaque parti se rassemble sur la base d'un programme et de cela s'ensuit que, dès qu'un programme est écrit, le parti qui devrait être lié par ce programme, doit encore se former $»^{1}$. Journaliste au sein de quotidiens sociaux-démocrates dans les années 1890, Adolf Braun (1862-1929)² publie, en 1891, un livre qu'il présente comme la première contribution destinée à décrire les partis allemands et leurs programmes politiques. Ce recueil social-démocrate répond aux intérêts pratiques des professionnels de la politique en mettant à plat l'offre politique. Braun, membre de commissions programmatiques du SPD $(1921,1925)$, destine son ouvrage aux " praticiens de la politique » qui doivent " prendre le contenu des programmes comme point d'attaque dans la lutte politique $»^{3}$. Après lui, en 1907, Felix Salomon, professeur d'histoire à l'Université de Leipzig, produit un ouvrage en deux volumes se présentant comme « la première synthèse systématique et objective " (non partisane) de l'ensemble des programmes politiques allemands de 1844 au début du $20^{\mathrm{e}}$ siècle ${ }^{4}$. Avant d'être une histoire des programmes, cet ouvrage reste une histoire des idées politiques par les textes. Felix Salomon ordonne les programmes par «courants de pensée » (conservatisme, libéralisme, catholicisme politique, socialisme). Les programmes partisans figurent, au milieu d'autres types de textes, parmi les expressions de ces courants - ainsi, de multiples articles extraits de revues intellectuelles du $19^{\mathrm{e}}$ siècle font figure de "programmes".

Les manuels produits dans la lignée de Friedrich Salomon pour rassembler les programmes des partis recueillent des textes très disparates au $19^{\mathrm{e}}$ siècle (des lettres, des articles de revue ou de journaux, des discours) alors que, pour la période de Weimar, les programmes colligés sont assez semblables dans leur forme et dans leur désignation ${ }^{5}$. L'existence d'une culture programmatique objectivée, différemment selon les partis, dans la publication de manuels, dans la spécialisation de «bureaux » partisans ou dans les pratiques de diffusion ou d'archivage des organisations, rend possible l'existence d'un " horizon d'attente » ${ }^{6}$ programmatique.

Cette standardisation est liée à la généralisation des programmes partisans. Avec leurs programmes, les partis se donnent à voir comme unité. Un élément significatif de ce phénomène est la disparition des signatures individuelles, souvent accompagnées d'indications sur le statut social (profession, adresse), qui ornent, parfois par centaines, les programmes des comités électoraux ${ }^{7}$. La " triade parti-programme-investiture " ${ }^{8}$ devient une conception commune, et rend superflues ces signatures individuelles pour l'ensemble des organisations. Pour le SPD, nous n'avons pas retrouvé de trace de programmes endossés par de nombreuses personnes en raison de l'ancienneté de l'organisation et de sa faible dotation en "notables». En revanche, le Parti national-libéral, un des grands partis (notabiliaire) du système impérial,

\footnotetext{
1. Paul de Lagarde, Programm für die konservative Partei, Göttingen, Dietrichsche Verlagsbuchhandlung, 1881, p. 1 (nous soulignons).

2. Ce juriste d'origine autrichienne devient, dans les années 1920, I'un des dirigeants du SPD dont il est député au Reichstag (1919-1929), secrétaire national de 1920 à sa mort et dont il préside les commissions programmatiques de 1921 et 1925.

3. Adolf Braun, Die Parteien des deutschen Reichtags. Ihre Programme, Entwicklung und Stärke, Stuttgart, Dietz, 1893, p. 5.

4. Felix Salomon, Die deutschen Parteiprogramme, Leipzig, Teubner, 1907, 2 t.

5. Wilhelm Mommsen, Günther Franz, Die deutschen Parteiprogramme, Leipzig, Teubner, 1932, 3 vol.

6. H. R. Jauss, Pour une esthétique..., op. cit.

7. On renvoie au manuel de F. Salomon, Die deutschen Parteiprogramme, op. cit.

8. Michel Offerlé, "Le nombre de voix. Électeurs, partis et électorat socialistes à la fin du XIXe siècle en France ", Actes de la recherche en sciences sociales, 71-72, 1988, p. 5-21, dont p. 11.
} 
marque bien cette tendance à la dépersonnalisation du programme. C'est en 1913 que le parti endosse son premier appel électoral sans mention de signataires individuels ${ }^{1}$. Cet endossement en nom collectif est rendu possible par la diffusion du congrès comme technique de production de la parole collective. Les congrès des partis politiques, dans leur variabilité historique $^{2}$, ou les procédures d'adoption des programmes sont des objets importants de l'analyse : ils sont des manières de faire exister et rendre visibles "le parti »" Ce sont bien les congrès qui transforment les programmes partisans, de projet des commissions programmatiques ou des dirigeants en un programme partisan. Les programmes finissent par faire parler le parti d'une seule voix, cela autant que de le faire exister de manière indépendante de ses incarnations individuelles.

La standardisation n'empêche pas des variations interorganisationnelles : le SPD se singularise par une autonomisation intrapartisane de spécialistes des discours programmatiques (les «théoriciens $»)^{4}$. Ses programmes acquièrent ainsi des caractéristiques spécifiques: la " ligne », appelée programme du parti, est une production de la centrale partisane qui mobilise ces théoriciens. L'une des dimensions les plus caractéristiques de ces programmes sociauxdémocrates est l'importance du « diagnostic social» (Zeitanalyse) que ces théoriciens rédigent en s'appuyant sur un savoir de parti spécifique, la «science marxiste ». Dans le programme de 1925, cette analyse, nommée dans le commentaire officiel "partie fondamentale », est suivie par des revendications qui portent le nom de "programme d'action », ce qui explique que le programme de Bad Godesberg soit «fondamental », puisqu'il était censé s'articuler à un «programme d'action » adopté en 1954. Un programme du parti reste en vigueur plusieurs années : de fait, de 1891 à aujourd'hui, six programmes de ce type ont été adoptés. Ce type de programme est donc distinct des programmes de gouvernement ou des programmes électoraux - notamment en raison du caractère général des formulations. Les spécialistes de la théorie produisent des brochures bon marché commentant les programmes, et avalisées par le comité directeur du SPD.

\section{La matérialité : rédacteurs et rédaction du programme de 1959}

Forme de texte inscrite au "répertoire des technologies partisanes " ${ }^{6}$, le programme reste le "produit d'une activité humaine passée [qui] n'existe que dans la mesure où l'activité humaine continue à le produire $»^{7}$. Comment s'organise dès lors cette rencontre avec les textes du genre, et pourquoi les acteurs en font ils usage ? Une telle question se pose en particulier, dans notre cas, pour le programme de 1959. En effet, les théoriciens sociauxdémocrates constituent un personnel relativement stable du $19^{\mathrm{e}}$ siècle aux années 1920 : la stabilité du genre est en quelque sorte garantie par la continuité de ce personnel qui l'invente, Eduard Bernstein ou Karl Kautsky en étant les exemples les plus connus. Leur rôle est central dans les commissions programmatiques des années 1890 aux années 1920. Hermann

\footnotetext{
1. Programmatische Kundgebungen der Nationalliberalen Partei, 1866-1913, Berlin, Reichsverlag, 1913, p. 127.

2. Alain Bergounioux, Frédéric Sawicki, Pierre Serne, "L'objet "congrès socialiste" en débat ", Recherche socialiste, 12, septembre 2000.

3. Christophe Prochasson, "Jaurès en congrès ou l'utopie délibérative », Société d'études jaurésiennes. Cahiers Jaurès, 187-188, 2008, p. 63-85.

4. T. Welskopp, Das Banner der Brüderlichkeit..., op. cit., p. 50-51.

5. Karl Kautsky, "Grundsätzlicher Teil », dans Das Heidelberger Programm. Grundsätze und Förderungen der Sozialdemokratie, Berlin, Vorstand der Sozialdemokratischen Partei Deutschlands, 1925, p. 5-26.

6. Michel Offerlé, Les partis politiques, Paris, PUF, 2012 (Que sais-je ?), p. 36.

7. Peter Berger, Thomas Luckmann, La construction sociale de la réalité, Paris, Mouton, 1985, p. 115.
} 
Molkenbuhr (1858-1927) est un autre exemple: cet ouvrier du tabac devient un expert reconnu en statistique sociale et est député de 1890 à 1924. Il est membre des quatre commissions de rédaction du programme réunies entre 1875 (programme de Gotha) et 1925, et préside la commission de 1921. À l'inverse de cette continuité du personnel, le programme de 1959 est rédigé par un personnel entièrement renouvelé.

Les rédacteurs du programme de Bad Godesberg font du corpus des programmes sociauxdémocrates du passé un élément central de leur travail. Dès la constitution de la commission, le responsable des questions culturelles au sein du Parti envoie du «matériel » à ses membres, charge à eux de l' « étudier »'. Ce matériel est composé de l'ensemble des programmes du SPD, à commencer par le Manifeste du Parti communiste. Le rapporteur de la commission programmatique (et responsable des questions programmatiques pour le comité directeur du SPD) Willi Eichler (1896-1971) $)^{2}$ indique, dans une préface à un recueil de programmes republié en 1958, que «pour la discussion de ce projet [de Stuttgart], connaître les conceptions qui ont guidé les programmateurs antérieurs de la social-démocratie nous semble être particulièrement utile puisqu'ils se sont objectivement toujours trouvés affrontés aux mêmes questions $»^{3}$.

Disposer des textes ne conduit pas nécessairement à en faire usage. Cependant, les archives montrent que les membres de la commission les lisent, la participation au travail des commissions en impliquant la maitrise. Wolfgang Abendroth commente ces programmes dans ses travaux ${ }^{4}$. De la même manière, le président de l'association allemande de sociologie Otto Stammer (1900-1978), autre membre de la commission, en fait la base d'un séminaire à la Freie Universität de Berlin ${ }^{5}$. Plus généralement, les programmes antérieurs sont cités sur des documents déposés aux archives, en notes marginales, mais aussi décomposés suivant la structure théorie/revendications qui est celle guidant leur rédaction. Le juriste et député au Bundestag Walter Menzel (1901-1963), dans une lettre circulaire du 6 mai 1955 aux membres de la commission économique du Parti indique :

«Qui lit attentivement les comptes rendus des congrès du Parti, surtout celui du congrès de Görlitz [1921] me donnera raison lorsque je prétends que nous n'arriverons pas, dans le programme partisan que nous soumettrons dans l'un des prochains congrès à une situation comparable à celle de Görlitz par exemple. » ${ }^{6}$

La lecture des programmes et des comptes rendus des congrès est attestée par d'autres indications. Manuscrites au dos d'un texte, des notes de Menzel se réferent à une réédition partisane de 1947 du programme de Görlitz et aux commentaires en congrès qu'inclut cette réédition ${ }^{7}$. Ces notes reprennent le matériel distribué aux membres de la commission avec des numéros de page précis : « comp. Heidelberg p. 19 » ou à «Heidelberg I » (référence à la partie théorique du programme de 1925$)^{8}$.

1. Bonn, AdsD, Walter Menzel, R42.

2. Willi Eichler est un ancien employé de commerce formé à la théorie par le militantisme.

3. Willi Eichler, "Vorwort », dans SPD-Unterbezirk Düsseldorf-Mettmann. Programme der deutschen Sozialdemokratie von 1863 bis 1925, Düsseldorf, 1958, p. 5.

4. Wolfgang Abendroth, Aufstieg und Krise der deutschen Sozialdemokratie, Cologne, Pahl-Rugenstein, 1978

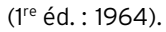

5. Bonn, AdsD, Otto Stammer, 035/7, cours d'Otto Stammer (Übung : Demokratischer Sozialismus und Marxismus, 1960).

6. Bonn, AdsD, Walter Menzel, R42.

7. Sozialistische Dokumente. Das Görlitzer Programm, Offenbach, Bollwerk Verlag, 1947.

8. Bonn, AdsD, Walter Menzel, R42. 
Fritz Sänger (1901-1984) est un instituteur devenu journaliste grâce à sa participation à la presse syndicale puis directeur de l'agence de presse ouest-allemande jusqu'à son licenciement au printemps 1959. Il est le principal rédacteur de la version finale du programme. Initialement sans "compétence exégétique »', Fritz Sänger se socialise à l'histoire programmatique de la social-démocratie au cours des quelques mois (de mai 1959 à novembre 1959) que dure sa mission de "correction» du programme. Dans l'entretien qu'il a avec Erich Ollenhauer au sujet de son éventuelle participation au travail de correction du projet, Sänger commence par affirmer son incompétence scientifique :

" Je ne possède aucune base scientifique. Les obligations quotidiennes m'ont toujours ramené au travail politique et syndical. Aussi n'ai-je rien pu dire ou écrire d'important sur la théorie du mouvement ouvrier. $»^{2}$

Cette déclaration montre que Sänger perçoit la maîtrise théorique comme un fondement du travail programmatique. À partir de mai 1959, Sänger se plonge dans la lecture des anciens programmes et de leurs commentaires ${ }^{3}$. Cette connaissance de la littérature lui est nécessaire puisqu'il doit justifier ses choix de réécriture du programme, ainsi auprès de Benedikt Kautsky $(1914-1960)^{4}$ :

\section{«Cher Camarade Kautsky,}

Je suis réellement navré de ne pas avoir pu respecter les délais qui avaient été prévus pour l'envoi du projet de programme fondamental. La quantité de travail était simplement trop grande et je n'ai pas pu terminer plus vite. [...]

De manière à mieux comprendre le contexte général, j'ai lu - autant qu'ils m'étaient accessibles et lisibles dans des délais brefs - d'anciens commentaires du programme d'Erfurt et d'Heidelberg. Ils m'ont conforté dans la conviction de laisser de côté beaucoup, sinon l'essentiel, des réflexions des commissions du Parti. II s'agissait en effet de commentaires et non d'énoncés programmatiques. Cela vaut en particulier pour la partie consacrée à l'ordre étatique où des conceptions de nature presque explicitement théorique sont discutées mais où ne sont pas faits des déclarations ou des constats précis. $»^{5}$

L'enjeu est de se faire reconnaître comme un individu pouvant légitimement (à ses yeux d'abord) manipuler le bien symbolique qu'est le programme ; et cette légitimation le conduit à effectuer un travail sur les textes programmatiques antérieurs.

\footnotetext{
1. Le terme de «compétence exégétique » est repris de Mohamed Tozy. Ce dernier montre que les rapports entre Islam et politique au Maroc sont construits autour de la production de sens religieux et d'appel à une tradition qui excluent les non-religieux de la construction du système religieux (p.166) et renforcent la position d'associations religieuses ou de fonctionnaires religieux au sein du champ politique. II labellise ces stratégies de construction de sens de "compétence exégétique" (p. 235): Mohamed Tozy, Monarchie et Islam politique au Maroc, Paris, Presses de Sciences Po, 1999. Cf. aussi la sociologie religieuse wébérienne et le contrôle par les brahmanes de l'accès aux Veda qui est la base d'« un monopole sur le savoir qui exclut » (Isabelle Kalinowki, " "Ils ne songent pas à désirer le nirvana". La sociologie des intellectuels dans Hindouisme et bouddhisme de Max Weber ", dans Johan Heilbron, Rémi Lenoir, Gisèle Sapiro (dir.), Pour une histoire des sciences sociales. Hommage à Pierre Bourdieu, Paris, Fayard, 2004, p. 181-201, ici p. 188-189.
}

2. Bonn, AdsD, Fritz Sänger, 53.

3. Entre autres: le programme d'Heidelberg, le Manifeste communiste dans une édition moscovite de 1945, les textes programmatiques publiés en 1946 par le SPD (Erfurt, textes de Lassalle), le projet de 1958, le manifeste de Prague de 1934 (Bonn, AdsD, Fritz Sänger, 324).

4. Il s'agit du fils de Karl Kautsky. Docteur en économie, il devient enseignant à I'université en Autriche après son retour de camp de concentration. Il est membre de la seconde commission programmatique du SPD nommée en mai 1959.

5. Bonn, AdsD, Fritz Sänger, 53, lettre à Benedikt Kautsky du 22 juin 1959. 


\section{Les « conventions notoires » : I'exemple du « préambule » du programme de 1959}

Bad Godesberg marque cependant, à plusieurs titres, une rupture formelle dans l'histoire des programmes sociaux-démocrates. Les analyses d'Hans Robert Jauss construisent la notion socio-littéraire de genre pour mettre en évidence les ruptures formelles à l'origine des «scandales» et des révolutions littéraires ${ }^{1}$. Les «conventions notoires» régissant l'écriture d'un programme sont interprétées en fonction des intérêts des acteurs et de leur capacité à s'imposer dans des interactions. En 1959 est abandonné le traditionnel diagnostic social (la première partie des programmes depuis 1891). Malgré l'opposition des scientifiques de la commission, le comité directeur accepte de réduire le «diagnostic social» aux dimensions d'un bref «préambule » littéraire du programme (cf. illustration), qui n'a pas de nom stable (introduction, poème, préambule).

La défense du « diagnostic social » s'appuie précisément sur les règles du genre programmatique, et donc, le caractère déroutant du préambule produit pour remplacer ce diagnostic. Wolfgang Abendroth le dénonce «comme l'un des énoncés politiques les plus étranges qu'il [lui] ait été donné de lire dans toute [sa] vie »². En 1978 (quinze années après l'adoption du programme), un autre membre de la commission, Gerhard Weisser (1898-1989), professeur de politique sociale à Cologne, demande raison de la suppression de l'analyse de la société à Fritz Sänger, le rédacteur de la version finale du projet de programme :

"Votre commission de rédaction a supprimé sans la remplacer notre proposition de développements pour le diagnostic social entre le congrès de Stuttgart [1958] et le congrès de Bad Godesberg [1959]. Je n'ai jamais pu m'expliquer, même à l'époque, cette suppression sans remplacement. Je n'ai pu que me dire que votre commission trouvait le projet de Stuttgart trop long et a considéré que I'on pouvait se dispenser du diagnostic. Je me suis donné pour principe de ne pas faire d'opposition dans un congrès: les congrès sont toujours passionnés. Mais la chose avait pour moi, à l'époque, une signification certaine et cette signification n'a fait que grandir.

À l'époque cela a signifié pour moi l'échec d'une action dont Kurt Schumacher [président du SPD jusqu'à sa mort en 1952] m'avait fait sentir l'importance. II avait insisté pour que le programme contienne un développement qu'il appelait "axiomatique" et que le programme a appelé "valeurs fondamentales". II m'a fait pour ainsi dire promettre que je devais tout faire pour que ne manque pas au programme le développement historico-théorique et analytique que les programmes ont contenu depuis cent ans. $»^{3}$

Dans ce texte, Weisser ne considère pas que le préambule poétique puisse être considéré comme remplaçant l'analyse rationnelle de la société. Il mobilise la tradition du genre pour le contester: le scandale de forme du programme de Bad Godesberg signale le contenu normatif des programmes, de ce « que les programmes ont contenu depuis cent ans », objectivé dans le corpus des textes anciens. Ces tensions sur le contenu du texte invitent à explorer les dynamiques de sa fabrication en cassant l'image d'unité que le vote du programme par un congrès donne à son auteur.

\footnotetext{
1. H. R. Jauss, "Histoire et littérature», art. cité, p. 58.

2. Wolfgang Abendroth, Ein Leben in der Arbeiterbewegung, Stuttgart, Suhrkamp, 1976, p. 249.

3. Bonn, AdsD, Fritz Sänger, 59, lettre de Gerhard Weisser à Fritz Sänger du $1^{\text {er }}$ août 1978.
} 


\section{Illustration. Le "préambule» du programme de Bad Godesberg (traduction officielle de 1960)}

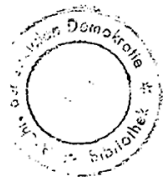

A $89-3513$
Publié par le Friedrich-Fbert-Stiftung

53 Bonn-Bad Godesberg, Kölner Straße 149
Imprimé au Vorwärts-Druck, 53 Bonn-Bad Godesberg
Telle est la contradic lion de notre temps que l'homme a libere los forces originaires de l'alome el qu'i! s'ellraie raintentant des conséquences;

que l'homme a développé les torces productives au plus haut degrê, a accumulé des richesses incommensurables sans donner à lous une part équitable de cette entreprise commune;

que J'homme s'est assujerti les espaces de cette terze, qu'il a rapproché les continents, mais gue, malgré tout, des olocs de puissance armés jusqu'aux dents séfarent les peuples, les uns des autres, plus que jamais et que des systemes totalitaires menacent la liberté de l'homme.

C'est pourquoi l'homme, averti par les guerres de destruction et la barharie d'un passé très récent, a peur de son propre avenir, parce grien tout instant, en tout lieu du monde, la carence humaine peut déclencher le chaos de l'aulodestruction.

Mais c'est aussi l'e spoir de ce temps, que l'homme puisse, a làge atomique, alléger son existence, se libérer de soucis et créer le bien-être pour lous, s'il utlise sa domination tous les jours croissante sur les forces de la nature à des fins exclusivement pacifiques; que l'homme peut assurer la paix mondiale, s'il-rentorce l'ordre juridique international, réduit la métiance entre les peuples et empèche la course aux armements;

Que lhomme puisse alors, pour la première lois dans son histoire, assurer à chacun l'épanouissement de sa personnalité dans une démocratie consolidée en vue d'une vie ziche sur le plan eullure! et Jibérée de la misère el de la peur.

C'est pour résoudre celte contradiction que nous nous sentons appolés en tant qu' hommes. C'est en ros mains que téside la responsabilité dagir pour un avenit teureux ou pour rautodestruc. tion du gente humain.

Ce' n'est que par une nouvelle et meilleure organisation de la société, que Ihomme peut se frayer un chemin vers la liberte.

Celle nouvelle et mellieure orgenisation constilue jobjectlf du socialisme démocratique.

\section{Division du travail collectif et modes de scientifisation du social}

$\mathrm{Q}$

ui est l'auteur du programme de Bad Godesberg? Le 23 décembre 1966, Fritz Sänger, rédacteur du projet soumis au congrès, écrit à Willi Eichler pour déplorer qu'un journal ait fait de lui « le père du programme ». Le journaliste, qui rédigeait un article à l'occasion du $65^{\mathrm{e}}$ anniversaire de l'ancien directeur de l'agence de presse allemande (dpa), aurait, dit-il, commis une erreur et ce serait bien à Willi Eichler que reviendrait d'avoir posé les fondements intellectuels du programme ${ }^{1}$. Depuis les années 1970 surtout, les participants au travail programmatique, les biographes et les journalistes multiplient les assignations auctoriales sur le programme. Eichler est, souvent, considéré comme le «père du programme de Bad Godesberg »'. L'ouvrage de Harmut Soell sur Fritz Erler $^{3}$ ouvre la voie à un conflit de paternité en promouvant la revalorisation du " rôle de Fritz Erler» dans la production de Bad Godesberg. La presse conteste, en effet, à partir des remarques de Soell, l'importance de celui d'Herbert Wehner ${ }^{4}$. Masaaki Yasuno entend, pour sa part, réhabiliter le rôle d'Erich Ollenhauer dans la production du programme 5 .

1. Bonn, AdsD, Fritz Sänger, 59, lettre à Willi Eichler du 23 décembre 1966.

2. Thomas Meyer, «Willi Eichler, Vater des Godesberger Programms. Eine Erinnerung zum 20. Todestag ", Neue Gesellschaft. Frankfurter Hefte, 38 (11), 1991, p. 1048-1049.

3. Hartmut Soell, Fritz Erler. Eine politische Biographie, Bonn, Dietz, 1976.

4. "Stehen und fallen mit der roten Fahne ?", Der Spiegel, 23 août 1976.

5. Masaaki Yasuno, Die Entwicklung des Godesberger Programms und die Rolle Erich Ollenhauers, Bonn, Schriftreihe der Friedrich-Ebert Stiftung, 2010. 
Les développements sur l'ordre étatique (staatliche Ordnung) font l'objet d'un conflit d'attribution entre historiens ${ }^{1}$. Cela vaut également pour d'autres développements économiques du programme, attribués par les exégètes à Karl Schiller ou à Heinrich Deist ${ }^{2}$. L'importance de Benedikt Kautsky est souvent signalée ; elle n’en reste pas moins contestée, par exemple par Fritz Sänger ${ }^{3}$.

Ce jeu des assignations découle d'une illusion héroïque ${ }^{4}$ cherchant à mesurer l'« influence » d'un individu sur le texte programmatique en n'explorant pas la complexité des liens qui unissent les individus et l'œuvre ${ }^{5}$. Ces interrogations sur le « rôle » des rédacteurs équivalent à des tests de paternité qui manquent rarement d'être contestés. Ce problème découle d'une conception individualiste de la production des idées politiques. Autour de Lucien Febvre appelant à isoler la théorie politique de l'histoire et à libérer cette dernière des impossibles quêtes de paternité ${ }^{6}$, l'histoire des mentalités avait formé son programme de recherche en récusant «avec constance les notions d'influence, de filiation et d'engendrement ${ }^{7} »$. Notre recherche sur les programmes n'entend pas projeter sur les rédacteurs du programme ces catégories propres à la critique d'art, tournant autour de l'individualité créatrice. Il s'agit de replacer l'ensemble des acteurs et des dispositifs de production dans une sociologie de l'organisation. Pour reprendre les mots de Karl Mannheim, «la conception individualiste du problème du savoir donne ainsi de la pensée collective une image aussi fausse que si, dans une usine hautement spécialisée de 2000 ouvriers, on imaginait la technique, le mode de travail et la productivité de telle manière que chacun de ces 2000 ouvriers travaillerait dans une pièce isolée, procédant pour soi-même et au même moment aux mêmes opérations et fabriquant lui-même chaque pièce de $\mathrm{A}$ à $\mathrm{Z} »^{8}$.

Établir les logiques sociales à l'œuvre dans la production programmatique revient à donner chair aux acteurs qui produisent, matériellement et socialement (en leur transférant du crédit social), les textes. Aussi les «commissions» ou les «équipes» en charge du programme sont-elles des observatoires de deux phénomènes intimement liés : l'organisation et la division du travail internes du parti, d'une part, et, d'autre part, les transactions intersectorielles, et donc des liens établis sous des formes plus ou moins objectivées et routinisées entre des groupes mobilisés, des organisations appartenant au "réseau de consolidation » ${ }^{9}$ du parti, des spécialistes partisans et les acteurs formellement en charge de la production d'un texte. Une commission programmatique ou une "équipe » en charge du programme, avec ses

1. Martin Wieczorek, "Martin Drahts Rolle bei den Beratungen über das Godesberger Parteiprogramm der SPD ", dans Michael Henkel, Oliver Lembcke (Hrsg.), Moderne Staatswissenschaft. Beiträge zu Leben und Werk Martin Drahts, Berlin, Duncker \& Humblot, 2010, p. 177-195; Dieter Gosewinkel, Adolf Arndt, Die Wiederbegründung des Rechtsstaats aus dem Geist der Sozialdemokratie (1945-1961), Bonn, Dietz, 1991.

2. Franz Barsig, "Freiheit und Sozialismus. Der lange "Marsch" der SPD nach Godesberg ", dans Roderich Klett, Wolfgang Pohl (Hrsg.), Stationen einer Republik, Stuttgart, dva, 1979, p. 93-110, ici p. 98.

3. Bonn, AdsD, Fritz Sänger, 59, lettre de Wedigo de Vivanco (doctorant en histoire italien) à Fritz Sänger du 6 janvier 1976 (notes marginales de Sänger).

4. Michel Dobry, Sociologie des crises politiques, Paris, Presses de Sciences Po, 1992 (1re éd. : 1986), p. 79 et suiv. 5. «Lorsqu'on pense en termes d'influence, on perd une bonne partie de ces nuances, et l'analyse s'appauvrit faute de moyens" (Michael Baxandall, Formes de l'intention. Sur l'explication historique des tableaux, Paris, Éditions Jacqueline Chambon, 1991 (1 $1^{\text {re }}$ éd. étr. : 1985), p. 107).

6. Lucien Febvre, "Une question d'influence. Proudhon et le syndicalisme contemporain », Revue de synthèse, 19 (2), 1909, p. 179-193, ici p. 191.

7. Philippe Poirrier, Les enjeux de I'histoire culturelle. L'histoire en débat, Paris, Seuil, 2004, p. 148.

8. Karl Mannheim, Idéologie et utopie, Paris, Éditions de la MSH, 2006 (1 éd. : 1929), p. 24.

9. M. Dobry, Sociologie des crises politiques, op. cit., p. 110. 
auditions et solliciteurs divers ${ }^{1}$, constituent, à cet égard, des espaces de transactions collusives multisectorielles ${ }^{2}$. Ces transactions permettent aux représentants de différents secteurs sociaux de faire reconnaitre leurs intérêts sectoriels comme des enjeux programmatiques.

\section{La scientifisation du politique}

La production des programmes est sensible aux modes de «scientifisation du social $»^{3}$, c'està-dire à l'intervention croissante des experts, aux modalités que ces interventions peuvent prendre, et aux manières dont sont utilisés leurs arguments ou les résultats de leurs recherches en politique. Si ce diagnostic de scientifisation du social a été posé par les historiens pour une période courant depuis le $19^{\mathrm{e}}$ siècle et si le rôle des sciences sociales ne s'était pas démenti avec le régime national-socialiste ${ }^{4}$, les débuts de la République fédérale sont ceux d'une « dépolitisation » du politique et d'une participation accrue des praticiens des sciences sociales ${ }^{5}$ (et, parmi eux, notamment les économistes ${ }^{6}$ ) à la production des politiques dans les multiples arènes constituées autour du gouvernement fédéral. Comme le montre Gabriele Metzler, la politique, en République fédérale, a une tendance à être confiée à des conseils scientifiques après 1945, en particulier sous l'effet de la participation des économistes ordolibéraux à l'exercice du pouvoir d'État. La spécificité de l'analyse du SPD est de montrer la manière dont une dynamique partisane de scientifisation se construit en réaction à cette dépolitisation de la politique et de l'économie au niveau des institutions publiques étatiques. L'analyse des programmes pose la question des modalités d'intervention des différentes catégories d'experts, certaines étant constituées en réponse à des préoccupations politiques (à l'instar des spécialistes du marxisme ou de diverses formes des sciences socio-biologiques ou racistes $)^{7}$.

Les « experts» sont des produits de configurations socio-historiques précises ${ }^{8}$. En ce qui concerne le SPD, la montée des experts et la scientifisation du politique prend un tour très différent suivant que l'on observe les années 1920 ou les années 1950. En 1891, le programme d'Erfurt, premier programme du Parti après la fin de son interdiction, est relativement peu spécialisé. La théorie est le produit du travail de Karl Kautsky, alors que les revendications seraient de la plume d'Eduard Bernstein'. En 1921, la rédaction du programme procède d'un

\footnotetext{
1. Pour une description de la production des «injonctions " programmatiques dans une équipe de campagne contemporaine : Émilien Matter, Xavier Schmitt, "La société civile, entre devoir d'alerte et droit au chantage ", Slate, 3 avril 2012, 〈http://www.slate.fr/tribune/52579/societe-civile-alerte-chantage〉.

2. M. Dobry, Sociologie des crises politiques, op. cit.

3. On renvoie à l'article fondateur de Lutz Raphael, «Die Verwissenschaftlichung des Sozialen als methodische und konzeptionelle Herausforderung für eine Sozialgeschichte des 20. Jahrhunderts ", Geschichte und Gesellschaft, 22, 1996, p. 165-193.

4. Carsten Klingelmann, Soziologie und Politik. Sozialwissenschaftliches Expertenwissen im Dritten Reich und in der frühen westdeutschen Nachkriegszeit, Wiesbaden, VS Verlag, 2009.

5. Gabriele Metzler, Konzeptionen politischen Handelns von Adenauer bis Brandt. Politische Planung in pluralistischen Gesellschaft, Paderborn, Ferdinand Schöningh, 2005.

6. Alexander Nützenadel, Die Stunde der Ökonomen. Wissenschaft, Politik und Expertenkultur in der Bundesrepublik (1949-1974), Göttingen, Vandenhoeck \& Ruprecht, 2005.

7. L. Raphael, «Die Verwissenschaftlichung... », art. cité.

8. Cette diversité socio-historique de l'expertise politique en matière de production programmatique a été soulignée pour la France. Cf. Christian Le Bart, "Les partis politiques: quelle capacité programmatique ?", Les Cahiers français, 364, septembre-octobre 2011, p. 38-42; Philippe Zittoun, "Partis politiques et politiques du logement. Échanges de ressources entre dons et dettes politiques ", Revue française de science politique, 51 (5), octobre 2001, p. 683-706; Marie Ymonet, "Les héritiers du Capital. L'invention du marxisme en France au lendemain de la Commune ", Actes de la recherche en sciences sociales, 55, 1984, p. 3-14.

9. Ingrid Gilcher Holtey, Das Mandat des Intellektuellen. Karl Kautsky und die Sozialdemokratie, Berlin, Siedler Verlag, 1986.
} 
travail d'experts se partageant des thématiques. Lors de la préparation de ce nouveau programme, le théoricien du SPD Eduard Bernstein précise que la révolution, avec la socialisation de l'économie et du droit qu'elle a engendrée, conduit à la nécessité d'une «transformation de l'organisation du programme» vers un programme plus spécialisé ${ }^{\text {. }}$ D’après les notes manuscrites de Bernstein, dix comités spécialisés sont constitués : 1 / partie générale ; 2 / politique économique; 3 / questions financières ; 4 / politique sociale $; 5 /$ politique de santé ; 6/ questions juridiques ; 7/ politique culturelle ; 8/ questions constitutionnelles ; 9 / politique étrangère, union internationale et relations internationales ; 10/ question des femmes ${ }^{2}$. Ces comités donnent presque tous lieu à un chapitre dédié du programme, hormis la question des femmes et celle de la santé intégrées dans les développements sur la politique sociale. Ces comités sont constitués autour d'experts, formés pour l'essentiel à travers des activités militantes et salariées pour le parti et ses organisations satellites.

Pour n'en prendre qu'un exemple, Heinrich Cunow (1862-1936) participe à la rédaction de l'analyse générale. Heinrich Cunow a une carrière politique très typique de la social-démocratie jusqu'à sa bifurcation vers une carrière universitaire. Employé de commerce puis comptable passé par l'apprentissage, Cunow est journaliste au social-démocrate Hamburger Echo depuis le début des années 1890. Il devient collaborateur puis, en 1898, salarié de l'organe théorique du parti. Comme $76 \%$ des 41 membres des commissions programmatiques des années 1920, il a donc été salarié de la presse du Parti. Au sein de la revue théorique, Cunow se spécialise dans les travaux ethnologiques. Ses premières réflexions en la matière sont effectuées dans la lignée des questionnements d'Engels sur les origines de la famille et de l'État. Dans ce cadre, Cunow mobilise des sources espagnoles, apprend le quetchua et publie de nombreux textes sur les Incas. C'est également dans une discussion sur les stades de développement des sociétés humaines que Cunow mobilise des savoirs sur les sociétés primitives, allant des Indiens d'Amérique du Nord aux peuples australiens et aux Incas ${ }^{3}$. Après 1918, il devient député à l'Assemblée constituante et au Landtag de Prusse. Par la suite, bien que n'ayant pas de doctorat, il dirige le musée municipal d'ethnologie de Berlin et obtient un poste de professeur extraordinaire de Staatswissenschaften en 1919.

À cette première montée de l'expertise des années 1920, succède une expertise, appuyée sur des ressources externes au parti. En 1955, les spécialistes du programme sont essentiellement universitaires et l'expertise s'appuie sur des ressources déléguées par le champ scientifique. Après 1945, le système des organisations sociales-démocrates se constitue sur de nouvelles bases : le vivier important des rédacteurs des centaines de journaux du Parti se tarit alors que le SPD doit faire pièce à une expertise de professeurs massivement convoquée au service du gouvernement chrétien-démocrate de Konrad Adenauer. Dès la fin des années 1940, le SPD constitue 22 comités spécialisés (agriculture, économie, finances, droit, réunification, etc.) composés jusqu'aux deux tiers d'universitaires (enseignants, chercheurs ou docteurs) ${ }^{4}$. Entre 1920 et 1950 que le SPD bascule donc d'un modèle d'expertise partisan à une expertise

1. Cf. Adolf Braun, Das Programm der Sozialdemokratie. Vorschläge für eine Erneuerung, Berlin, Vorwärts, 1920, en particulier l'introduction et la contribution d'Eduard Bernstein: «Zur Frage eines neues Programms der sozialdemokratischen Partei Deutschlands", p. 24-33, ici p. 25.

2. Amsterdam, International Institute of Social History (IISG), fonds Bernstein, N2.

3. Heinrich Cunow, «Die Verfassung des Inkareichs", Die Neue Zeit, 14 (29), 1896, p. 75-81; Heinrich Cunow, Die Marxsche Geschichts-, Gesellschafts- und Staatstheorie, Berlin, Dietz, t. I et II, 1920 et 1921. L'ouvrage est parsemé de références à des peuples primitifs, y compris pour préciser ou critiquer les conceptions ethnologiques de Marx et Engels au nom de la vérité scientifique (t. II, p. 291 et p. 313-314, par exemple).

4. SPD Jahrbuch, 1958/1959, p. 249 et suiv. 
reposant sur des ressources déléguées par l'Université. Les membres des commissions programmatiques dans les années 1920, sont en effet très minoritairement universitaires. Alors que les rédacteurs des programmes adoptés dans les années 1920 sont à $21 \%$ diplômés de l'Université $(\mathrm{n}=41)$, ils le sont à $68 \%$ en $1955(\mathrm{n}=61)$, et les docteurs représentent une proportion de $14 \%$ en 1921 mais de $56 \%$ en 1955. En 1955, le président de la commission programmatique constate que "nous sommes obligés de prendre des gens qui ont étudié des questions spécifiques de manière approfondie et, de nos jours, comme tu le sais, cela va de plus en plus de pair avec l'exercice d'une profession académique ${ }^{1}$. Un membre du comité directeur du Parti va jusqu'à critiquer cette commission comme un " "conseil de professeurs" d'Eichler responsable d'un "texte presque illisible" " (le projet de programme de 1958).

Ces acteurs ne sont pas uniquement des scientifiques. Un programme se produit à l'intersection des dynamiques internes à l'organisation partisane et des liens de celle-ci avec les porte-parole d'intérêts externes lorsqu'ils existent. Le cas du programme de Bad Godesberg est à cet égard symptomatique : sa production coïncide, par exemple, avec l'orchestration par des organisations liées au parti d'une lutte contre "la mort atomique" (Kampf den Atomtod), c'est-à-dire contre la détention de l'arme nucléaire par la RFA. Walter Menzel dirige le comité interassociatif chargé d'organiser cette campagne. Il n'est donc pas étonnant que la première «crainte de notre époque » mentionnée dans le préambule du programme soit celle de la destruction par l'arme atomique (cf. illustration). De la même manière, d'autres associations sont présentes à travers des membres disposant de responsabilités en leur sein : syndicats (IG-Metall, représenté par son président, Otto Brenner, syndicats enseignants et étudiants), des représentants de groupes de travail interne au SPD ou d'associations de jeunesse, comme Lorenz Knorr, y sont représentés.

Cependant, même lorsqu'ils ne sont pas des scientifiques, ces acteurs restent sensibles au phénomène de scientifisation du social et de la politique. Ainsi Lorenz Knorr (né en 1921) se décrit lors de notre entretien comme un «intellectuel ouvrier». Imprimeur, il quitte la Tchécoslovaquie en 1945 et s'installe en Bavière et s'engage dans l'organisation du mouvement de jeunesse socialiste. Secrétaire fédéral de la Jeunesse socialiste (Sozialistische Jugend), il est un promoteur des "Républiques des enfants ", modèle éducatif appuyant le développement de la personnalité par une organisation rationnelle de l'espace, du temps et par l'esprit de communautés. Il fonde surtout un conseil scientifique de la Jeunesse socialiste, autour d'universitaires, pour donner une «base solide », c'est-à-dire « scientifique », au travail pédagogique pour dépasser la simple « représentation des intérêts » :

KF : J'ai aussi vu que vous étiez président du conseil scientifique de la Jeunesse...

LK : ... socialiste. Les choses étaient les suivantes. J'ai été à la présidence des Jeunesses socialistes et responsable pour la pédagogie. Et nous tombions sans arrêt sur des problèmes où nous nous disions que la discussion politique ne suffisait pas et qu'il fallait discuter les choses scientifiquement. Là, j'ai proposé de fonder un conseil scientifique avec une série de scientifiques, de professeurs qui ont travaillé avec nous et j'ai convoqué un cercle d'environ dix personnes, dix professeurs et docteurs; ils m'ont choisi comme manager, comme président quasiment de ce conseil et ils ont essayé de donner au travail politique de la pédagogie une meilleure base.

1. Bonn, AdsD, comité directeur - secrétariat Willi Eichler, 0 1699, lettre de Willi Eichler à Heinrich Albertz du 15 mars 1955.

2. Hartmut Soell, Fritz Erler. Eine politische Biographie, Bonn, Dietz, 1976, p. 323.

3. Lorenz Knorr, Moderne Zeltlagergestaltung, Francfort-sur-le-Main, Verlag Schaffende Jugend, 1957. 
KF : Et pouvez-vous décrire cette base? Quelle nouveauté pour cette base? Pourquoi était-ce important qu'il y ait des scientifiques qui contribuent à cette discussion?

LK : En ce qui concerne la politique, nous voulions en savoir plus sur les disciplines et sur la science de manière à ce que la politique ne soit pas une pure représentation des intérêts mais pour que tout tienne sur une base solide, et nous voulions fonder la pédagogie de telle manière que chacun d'entre nous a eu à s'occuper d'un scientifique déterminé. Moi-même, j'ai eu à m'occuper de Kurt Löwenstein', un pédagogue social-démocrate de la république de Weimar... ${ }^{2}$

Cette scientifisation a des effets sur l'organisation du travail programmatique lui-même.

\section{« Un programme n'est pas un texte de propagande »}

Le programme de Bad Godesberg donne une illustration de ces relations complexes, à la fois de déférence vis-à-vis des scientifiques qui écrivent seuls ou presque le premier projet du programme de 1959 et de défiance face à un texte que les dirigeants ne comprennent pas et dont ils ne maîtrisent pas le processus de production. Lorsque la commission d'universitaires soumet à la discussion un projet de programme en avril 1958, fruit de 4 années de travail, ce texte se voit critiqué pour sa complexité. Le comité directeur revendique alors sa modification non pas sur le fond (qui reste l'affaire des experts), mais sur la forme. En 1959, la direction nomme une commission restreinte dont l'unique objectif officiel est de "simplifier » le style et le langage du programme. Dans cette simplification se jouent bien les effets de la division du travail : les détenteurs du capital politique, membres du comité directeur, ont seuls le pouvoir de faire consacrer le texte comme programme. Cependant, ils n'ont pas, pour la plupart, de compétences spécialisées en économie, en droit ou en politique sociale. Dans la délégation de l'écriture du programme aux experts, ils se départissent donc d'une partie de leurs prérogatives pour asseoir la légitimité scientifique du programme.

Pour leur part, les universitaires font de la justesse technique des arguments une obligation. Juriste de formation, docteur et spécialiste des questions économiques du SPD, Heinrich Deist (1902-1964) défend dans une réunion de la commission économique du SPD la nécessité des développements techniques :

"Lorsque l'on dit que le projet n'est pas lisible pour l'homme de la rue, il ne s'agit pas d'un argument contre le programme. Ce programme est destiné en priorité aux permanents et aux membres intéressés du Parti et doit fournir à ces personnes des faits, des arguments et des raisonnements politiques pour la confrontation intellectuelle. Un programme n'est pas un texte de propagande. ${ }^{3}$

Heinrich Deist marque bien là le décalage entre les acceptions ordinaires du programme et ses significations socialement enracinées ${ }^{4}$. Que le programme ne soit pas un texte de propagande ne prend sens que lorsque l'on sait que le débat qui anime la commission programmatique est celui des fondements scientifiques du socialisme et que son programme fondamental n'est pas en première ligne destiné à l'homme de la rue : ce sont les experts et

1. Kurt Löwenstein (1885-1939) est docteur en philosophie en 1910. II devient socialiste durant la première guerre mondiale et adhère à I'USPD. Député au Reichstag en 1920, il est considéré comme un pédagogue important des mouvements d'éducation de la république de Weimar.

2. Entretien avec Lorenz Knorr, 31 juillet 2012.

3. Bonn, AdsD, Heinrich Deist, 44, compte rendu de la réunion du Comité à la politique économie du SPD (23 janvier 1959).

4. On trouverait un décalage identique en s'intéressant aux programmes des années 1920. L'objectif n'est pas de s'adresser à un électorat, mais d'élever la conscience culturelle des travailleurs: "Nos visions sont si spé- 
les militants aguerris qui en sont les «lecteurs implicites $»^{1}$. On comprendra également que le bureau de la propagande du Parti ne soit pas associé à la production du texte avant que les scientifiques aient remis leur copie, et que le comité directeur reprenne la main sur le programme. Généralement sans mandat et sans fonction de direction au sein du Parti, les experts du Parti sont peu associés aux opérations de «simplification » du texte qui ont lieu entre mai et septembre 1959, confiées à deux journalistes. Seuls les experts en politique sociale, qui se déclarent «alarmés » par le projet dès l'été2, effectuent un travail de mobilisation en amont du congrès et parviennent à faire voter des motions modifiant le texte soumis par le comité directeur.

Cette organisation du travail a un effet sur le programme; son analyse permet de rendre compte des relations qu'entretiennent au sein de l'organisation les différentes catégories d'acteurs spécialisés, les politiques partisanes étant « le résultat toujours incertain de multiples marchandages dont les protagonistes, jamais fixés une fois pour toutes, n'ont jamais totalement la maîtrise initiale ni le contrôle ultérieur ${ }^{3}$. La division des tâches entre experts et politiques rend problématiques les interactions entre les diverses catégories d'acteurs. Dans les années 1980, le président d'une commission programmatique déplore ainsi que certains dirigeants pensent "que le parti est composé de deux groupes de militants : ceux qui produisent des papiers et ceux qui les classent en souriant pour reprendre, paisiblement et sans rien changer, leur activité politique qui serait bien différente de ce que croient les producteurs de papier $»^{4}$. Les relations, de contrôle ou de conseil, de déférence ou de défiance, entre dirigeants et experts font donc, à un pôle, des experts des fournisseurs de savoirs ancillaires et des apologètes ${ }^{5}$ d'une ligne politique à la détermination de laquelle ils contribuent peu ou, à l'autre extrême, des " conseillers du prince ", éminence grise ou pivots de la crédibilité des programmes ${ }^{6}$.

Considérer les programmes comme observatoires des relations sociales nouées autour de leur production évite l'analyse des «fonctions » du programme pour faire de son analyse une étude du fonctionnement des partis, et donc des transactions entre le parti et la société et des relations que les diverses catégories d'acteurs (experts, politiques, communicants, etc.) entretiennent dans le cadre d'une division du travail partisan On signale donc notamment ainsi le caractère variable des procédés de fabrication des programmes et des manières de faire des lieux de production des programmes des interfaces avec le milieu partisan.

\footnotetext{
cifiques, si différentes des visions traditionnelles que personne ne peut les comprendre sans faire d'effort. [...] Personne ne pourra comprendre notre programme s'il n'est, jusqu'à un certain point, rendu familier de notre pensée, d'une manière ou d'une autre: par des lectures, en écoutant des discours dans des réunions ou par des discussions privées. Quels que soient les efforts qu'on consente, le programme ne sera jamais un entonnoir grâce auquel on pourrait, sans autre difficulté, verser le socialisme dans les cerveaux " (Karl Kautsky, Die proletarische Revolution und ihr Programm, cité par Albrecht Langner, «Introduction », dans Karl Kautsky, Texte zu den Programmen der deutschen Sozialdemokratie. 1891-1925, Cologne, Jakob Hegner, 1968, p. 19).

1. Wolfgang Iser, Der implizite Leser, Stuttgart, Suhrkamp, 1976.

2. Bonn, AdsD, Ludwig Preller, 44-93, lettre du 16 juillet 1959 à Walter Auerbach (copie).

3. M. Offerlé, Les partis politiques, op. cit., p. 71.

4. Erhard Eppler, Grundwerte für ein neues Godesberger Programm, Hambourg, Rowohlt, 1984, p. 8.

5. Jacques Lagroye, La vérité dans l'Église catholique. Contestations et restauration d'un régime d'autorité, Paris, Belin, 2006.

6. Frédérique Matonti, Intellectuels communistes. Essai sur l'obéissance politique. La Nouvelle Critique (1967-1980), Paris, La Découverte, 2005.
} 


\section{Les usages contextualisés des programmes : le programme et ses liseurs}

$\mathrm{L}$ es significations d'un programme politique sont avant tout produites par leurs usages sociaux. Un texte tel que celui de 1959 est le résultat de multiples bricolages. La cohérence du document, sa signification, telle qu'elle est impliquée dans les multiples significations données à "faire son Bad Godesberg ", est le produit de l'effort de structuration post hoc d'acteurs disposés à user du texte et à lui imposer une signification univoque. La signification sociale d'un texte repose moins sur l'intention de son auteur que sur les formes de l'«attention » que lui portent ses lecteurs, c'est-à-dire sur les types d'usage qu'ils sont disposés à en faire, et donc sur les interprétations, même minimales, qu'ils donnent au texte en s'y référant.

Si l'on peut parler de textes " "sans situation" en dépit du substrat historique qu'ils charrient ", il faut bien ajouter que les situations dans lesquelles ils prennent sens sont " composées par les liseurs au cours de chaque lecture » ${ }^{1}$. Ces « liseurs» ne sont pas uniquement des interprètes appointés par les partis, mais de multiples individus en position de parler avec autorité sur un texte. Benjamin Lemoine a bien montré, en ce sens, que l'usage des programmes politiques dépasse les sociations partisanes pour s'étendre à une multitude d'acteurs au sein du champ politique ${ }^{2}$. De son énonciation par un candidat à sa dénonciation par un think tank ou des organisations sectorielles (par exemple, les théologiens catholiques pour le programme du SPD), des notes de renseignements généraux ${ }^{3}$ à celles des conseillers du président de la République ${ }^{4}$, des propositions faites par un candidat aux anticipations de leurs effets ou aux « rappels » des promesses (y compris pour peser dans les arbitrages administratifs lors de l'élaboration des politiques publiques ${ }^{5}$ ), de l'usage pratique dans le cadre de la transaction électorale par des militants qui distribuent le programme à l'usage théorique dans le cadre d'enseignements universitaires : le programme existe de manières multiples.

\section{Une sociologie des usages des programmes}

Cette multiplicité d'usages est patente dans la carrière du programme de 1959. Les militants s'interrogent, à partir du brouillon de 1958, sur la complexité du programme et la difficulté de s'en servir pour " aller vers les travailleurs ». L'usage du programme étant lié à sa capacité à styliser l'identité politique d'un parti, on comprend qu'il puisse servir à qualifier une déviance par rapport à sa ligne (comme en témoigne l'exclusion de l'association étudiante SDS en 1961), «nul n'étant censé ignorer le programme $»^{6}$. Les usages du programme montrent d'ailleurs la manière dont cette stylisation de l'identité partisane a joué un rôle dans le déplacement constant de ses significations.

Il y a, en effet, un décalage entre l'expérience historique d'un événement par ses contemporains, et la mémoire de cet événement ${ }^{7}$. Nous avons montré ailleurs que le congrès de 1959 et les

1. W. Iser, L'appel du texte, op. cit., p. 58-59.

2. Benjamin Lemoine, "Chiffrer les programmes politiques lors de la campagne présidentielle de 2007. Heurs et malheurs d'un instrument ", Revue française de science politique, 58 (3), juin 2008, p. 403-431.

3. Pascale Goetschel, "Le programme commun, I'opinion publique et le pouvoir", dans D. Tartakowsky, A. Bergounioux (dir.), L'union sans unité, op. cit., p. 133-146.

4. Bernard Lachaise, "Les droites et le programme commun, 1973-1978 ", dans D. Tartakowsky, A. Bergounioux (dir.), ibid., p. 119-131.

5. Ph. Zittoun, «Partis politiques... », art. cité, p. 693.

6. M. Offerlé, Les partis politiques, op. cit., p. 94.

7. Bernard Pudal, Claude Pennetier, "Le congrès de Tours au miroir autobiographique », Le Mouvement social, 193, 2000, p. 61-87. 
commentaires officiels du programme sont inscrits dans une politique de la mémoire, particulière au $\mathrm{SPD}^{1}$. On entend insérer le programme dans la continuité de la pensée théorique du Parti, et s'en servir pour assurer la maintenance identitaire de l'organisation dans un congrès qui s'ouvre sur le constat qu' " au commencement de toute politique social-démocrate, il y a le Manifeste de $1848 »^{2}$ de Karl Marx alors que le commentaire officiel donné par Fritz Sänger multiplie les références aux théoriciens du passé, Marx, Engels, Kautsky ou Bernstein.

«Bad Godesberg » n'est pas ab ovo un « texte de référence ». L'historien du SPD Peter Lösche indique ainsi que «le célèbre programme de Bad Godesberg n'intéressait presque personne dans les années 1960 alors que dans les années 1970, seuls quelques gauchistes étaient gênés de s'y référer $»^{3}$. Approximation des usages du texte sur une scène sociale particulière, le graphique, construit à partir d'un comptage des références au texte dans deux hebdomadaires allemands entre 1959 et 2012, montre que la seconde moitié des années 1960, et, surtout, les années 1970 sont les périodes de plus forte mobilisation du programme alors que les niveaux de référence sont moins importants entre 1959 et 1966.

L'accroissement du recours au programme, et donc des usages à analyser, coïncide avec deux séries d'événements : d'une part, l'accès du SPD aux fonctions gouvernementales en 1966 et en 1969 lorsque Willy Brandt devient chancelier fédéral; d'autre part, l'augmentation des tensions intrapartisanes résultant du mouvement étudiant de 1968 et de la mobilisation politique intense des universités dans les années 1970. Cette augmentation de la mention journalistique au programme s'explique dès lors par son usage comme recours explicatif du changement de stratégie du SPD, devenu parti de coalition gouvernementale. "Bad Godesberg " joue le rôle d'un ordonnateur de sens, de prophétie, au sens de Brigitte Gaïti ${ }^{4}$ : les journalistes s'en saisissent pour expliquer la stratégie de coalition avec l'Union chrétiennedémocrate (CDU) endossée par le SPD en 1966 (c'est-à-dire expliquer qu'un parti prônant un ordre socialiste ait pu accepter la participation à un gouvernement bourgeois). Il est, surtout, l'effet de son usage agonistique au sein du Parti, ce que nous allons évoquer plus précisément.

\section{Une sociologie des significations en situation}

Mettre en évidence la temporalité de la réception est un préalable à la contextualisation des "positions de lecture» (reading positions) des communautés interprétatives ${ }^{5}$. La référence au programme atteint son apogée dans les années 1970, lorsque des "communautés interprétatives " concurrentes s'emparent du programme pour produire des déviances, c'està-dire entrent en lutte pour avoir autorité sur le texte. À gauche, les Jeunes socialistes (les Jusos) appellent à la réalisation du «nouvel ordre économique et social promis par le programme de Bad Godesberg " contre une politique du gouvernement de Willy Brandt jugée trop accommodante avec le capitalisme. Ainsi, Thomas van der Vring (né en 1937, enseignant

\footnotetext{
1. Karim Fertikh, «Bad Godesberg dans le langage social-démocrate en 1959 », Cahiers d'histoire. Revue d'histoire critique, 11, 2011, p. 137-151.

2. SPD, compte rendu du congrès de Bad Godesberg (Protokoll der Verhandlungen), p. 36.

3. Peter Lösche, "Is the SPD Still a Labour Party? From "Community of Solidarity" to "Loosely Coupled Anarchy" ", dans David E. Barclay, Eric D. Weitz (eds), Between Reform and Revolution. German Socialism and Communism from 1840 to 1990, Oxford, Berghahn Books, 1998, p. 532-545, ici p. 539.

4. Brigitte Gaïti, De Gaulle prophète de la Cinquième République (1946-1962), Paris, Presses de Sciences Po, 1998.

5. Martin Barker, "On Being Ambitious for Audience Research", dans Isabelle Charpentier (dir.), Comment sont reçues les œuvres ? Actualités des recherches en sociologie de la réception et des publics, Grenoble, Créapolis, 2006, p. 27-42, dont p. 33.
} 
Graphique. Références au programme de Bad Godesberg dans deux hebdomadaires politiques allemands (1959-2012, $n=615$ articles mentionnant le programme)

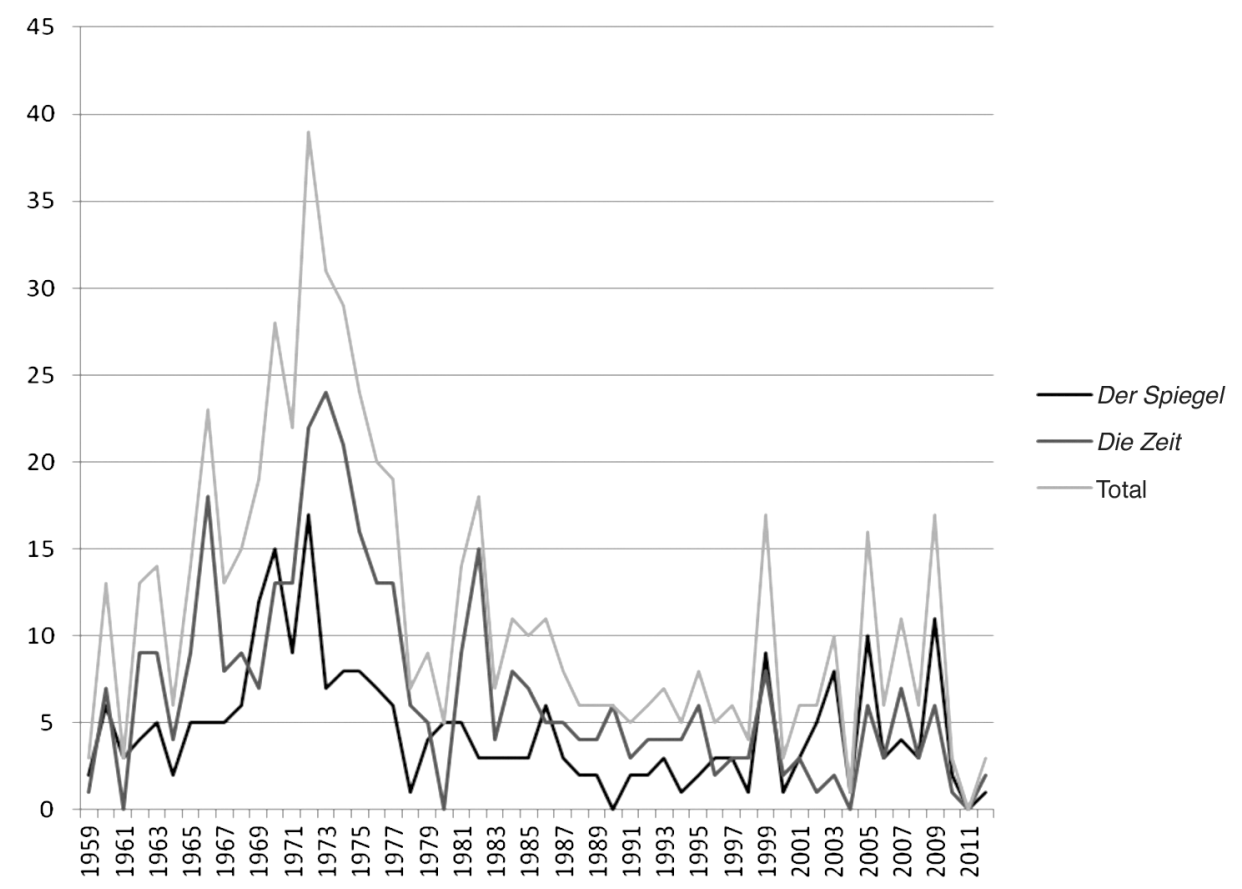

à la Technische Universität de Hanovre, membre de la présidence des Jusos en 1969) déplore qu' « il n'y ait rien à attendre de ce gouvernement dans la réalisation d'un ordre socialiste dans le sens de Bad Godesberg " ${ }^{1}$. À droite, la fragilisation des bastions de figures traditionnelles de la social-démocratie allemande provoquée par l'important renouvellement militant, amenant un tiers des militants de 1973 à appartenir aux Jusos, conduit à la structuration d'un courant réformiste dans le Parti. Ce courant, le cercle de Seeheim (originellement le cercle de Bad Godesberg), mobilise des philosophes et des spécialistes de sciences sociales, au sein de l'université aussi bien qu'au sein de la fondation du Parti, pour porter et diffuser une interprétation du programme de 1959 comme étant incompatible avec le marxisme ${ }^{2}$. Avec cette appropriation, si l'on veut, le programme a choisi un camp. Avec ces usages savants et politiques du programme, l'une des clefs de la carrière des théories politiques semble donnée : l'Université, en analysant, consacre aussi et contribue à faire "monter en théorie » ce qui n'est d'abord que prises de position et bricolages théoriques sur le champ.

Au-delà de cette « histoire allemande » du programme de Bad Godesberg, une histoire transnationale reste à écrire de la manière dont le texte franchit les frontières et dont ses médiateurs

1. Thomas van der Vring, "Schwein geschlachtet", Der Spiegel, 15 décembre 1969.

2. Le manifeste du cercle de Seeheim est consacré à cette démonstration sous le titre "Bad Godesberg et le présent " (Godesberg und die Gegenwart. Ein Beitrag zur innerparteilichen Diskussion über Inhalte und Methoden sozialdemokratischer Politik, Bonn, Verlag Neue Gesellschaft 1975). D'autres textes procèdent à la même labellisation, comme celui des philosophes, membres du cercle de Seeheim: Alexander Schwan, Gesine Schwan, Sozialdemokratie und Marxismus. Zum Spannungsverhältnis von Godesberger Programm und marxistischer Theorie, Hambourg, Hoffmann \& Campe, 1974. 
le ploient à des significations particulières pour l'introduire dans un nouvel espace de référence. Son arrivée en France est, ainsi, largement l'effet d'intellectuels de la «Deuxième Gauche » autour d'une réhabilitation de la social-démocratie ${ }^{1}$. De multiples ouvrages en font une référence de la social-démocratie essentiellement caractérisée par l'économie mixte et la concertation entre partenaires sociaux ${ }^{2}$ ou en proposant une synthèse entre social-démocratie et autogestion (comme dans Qu'est-ce que la social-démocratie?, ouvrage qui se conclut sur des extraits du programme de $1959^{3}$ ). Au-delà de ce travail d'importation, la référence à Bad Godesberg s'épure souvent de toute référence contextuelle, et le programme devient un mot de passe pour signifier la rénovation doctrinale nécessaire de parti de gauche (voire l'abandon de dogmes dépassés dans des partis de droite).

«Bad Godesberg» reste un texte exceptionnel. De nombreux programmes ne connaissent pas la gloire de l'accès au canon : le programme de 1989 du SPD succède ainsi au programme de Bad Godesberg en 1989. Cinq après son adoption, le président de la commission en charge de sa rédaction, Hans-Jochen Vogel, déplore que « le programme de Berlin soit traité au sein du Parti à peu près comme un document confidentiel $»^{4}$. De la même manière, et exception faite de quelques textes historiens, la célébrité du programme de l'Arche de la Défense du Parti socialiste français (1992) tient à l'oubli dans lequel il est tombé.

La sociologie des programmes politiques, telle que nous l'avons présentée dans cet article, a donc l'intérêt de faire des programmes l'observatoire de phénomènes politiques généraux, et non un sous-espace autonomisé du savoir disciplinaire. Ces orientations évitent la trop grande fascination du texte (à interpréter). Nos orientations rapatrient l'étude des programmes politiques dans la science sociale «normale », au sens de Thomas Kuhn. Il ne s'agit pas pour nous de prétendre offrir une alternative méthodologique au $C M P$, car ce type de recherche intensive est impraticable à grande échelle. Néanmoins, nous voulions ici montrer qu'il existe de nombreuses pistes de recherche fécondes à emprunter en matière d'analyse des programmes, et que celles-ci permettent de sortir du «no man's land» (pour reprendre une formule de W. Iser) de l'exégèse textuelle. En ce sens, nos références multiples aux travaux des sociologues, des politistes et des historiens relèvent d'une même volonté d'intégrer la sociologie des programmes dans une science sociale des textes, des organisations et des idées politiques. Cette sociologie des programmes vise une cumulativité fondée sur son lien étroit avec les sciences sociales du politique.

Cet article a cherché à retrouver, sous l'apparente évidence des programmes, leur dimension historiquement variable, et en particulier les effets différenciés de la mobilisation des experts au service du politique - de la scientifisation du politique. Produits de marchandages intrapartisans et de transactions avec la société, les programmes politiques deviennent, au prix des opérations de production et de sacre, des "fétiches", au sens où le sigle efface les conditions concrètes et individuelles de la production pour en faire une déclaration collective,

\footnotetext{
1. Christelle Flandre, Socialisme ou social-démocratie? Regards croisés français allemands, 1971-1981, Paris, L'Harmattan, 2006, p. 231 et suiv.

2. Entre autres: Alain Bergounioux, Bernard Manin, La social-démocratie ou le compromis, Paris, PUF, 1979.

3. Michel Rocard et al., Qu'est-ce que la social-démocratie?, Paris, Seuil, 1979 (Points politique).

4. Sozialdemokratischer Pressedienst, 15 décembre 1994.
} 
et dont l'usage produit les significations et la force contraignante pour des ensembles d'acteurs déterminés. L'intérêt est dès lors de les observer comme éléments de stratégies de consécration, comme révélateurs non pas des fonctions, mais des fonctionnements du parti. S'il y a bien autre chose à faire avec un texte que l'interpréter, comme le dit Gérard Genette, c'est qu'un texte est une manière de faire des choses et un support pour des relations sociales que les analyses contextualisées permettent de décrire ${ }^{1}$.

\section{Karim Fertikh}

Chercheur détaché au CNRS, Karim Fertikh est membre du Centre Georg-Simmel - Recherches francoallemandes en sciences sociales (UMR 8131, EHESS/CNRS) et est rattaché au laboratoire Savoirs, Acteurs, Gouvernement en Europe (UMR 7363, Université de Strasbourg/CNRS). Il est responsable du groupe de recherche sur les transformations de l'État social en Europe au sein du réseau franco-allemand «Saisir l'Europe/Europa als Herausforderung (MESR/Bundesministerium für Bildung und Forschung). Ses publications portent sur les partis, programmes et idées politiques, sur le militantisme et sur les politiques sociales en Europe. Il a notamment publié sur le même thème : "Le "nouvel ordre" du programme de Bad Godesberg. Sociologie d'une construction sociale de l'économie ", Lien social et politiques, 72 , automne 2014, p. 39-56; «Trois petits tours et puis s'en va... Marxisme et programme de Bad Godesberg du Parti social-démocrate allemand", Sociétés contemporaines, 81, mars 2011, p. 61-80 ; et «Bad Godesberg dans le langage social-démocrate en 1959 », Cahiers d'histoire. Revue d'histoire critique, 114, janvier-mars 2011, p. 137-151 (FMSH, 170 avenue de France, 75013 Paris, $<$ karim.fertikh@ehess.fr>). 


\section{Annexe. Tableau des producteurs du programme de 1959 cités dans l'article}

\begin{tabular}{|c|c|c|}
\hline Nom & $\begin{array}{l}\text { Profession, mandat ou poste partisan } \\
\text { (et niveau d'études) au moment } \\
\text { de la production du programme }\end{array}$ & $\begin{array}{l}\text { Fonction dans la production } \\
\text { du programme }\end{array}$ \\
\hline $\begin{array}{l}\text { Wolfgang Abendroth } \\
\text { (1906-1985) }\end{array}$ & Professeur de science politique, Marbourg & Membre de la $1^{\text {re }}$ commission \\
\hline $\begin{array}{l}\text { Heinrich Deist } \\
(1902-1964)\end{array}$ & $\begin{array}{l}\text { Membre du comité directeur, député } \\
\text { (docteur) }\end{array}$ & Membre des deux commissions \\
\hline $\begin{array}{l}\text { Willi Eichler } \\
\text { (1896-1971) }\end{array}$ & $\begin{array}{l}\text { Membre du comité directeur (aucun diplôme } \\
\text { universitaire) }\end{array}$ & $\begin{array}{l}\text { Rapporteur de la } 1^{\text {re }} \text { commission, } \\
\text { membre de la seconde }\end{array}$ \\
\hline $\begin{array}{l}\text { Fritz Erler } \\
(1913-1967)\end{array}$ & $\begin{array}{l}\text { Membre du comité directeur, député } \\
\text { (baccalauréat, aucun diplôme universitaire) }\end{array}$ & Membre de la $1^{\text {re }}$ commission \\
\hline $\begin{array}{l}\text { Lorenz Knorr } \\
\text { (1926) }\end{array}$ & $\begin{array}{l}\text { Président des Jeunes socialistes - les faucons } \\
\text { (Die Falken) }\end{array}$ & Membre de la $1^{\text {re }}$ commission \\
\hline $\begin{array}{l}\text { Walter Menzel } \\
(1901-1963)\end{array}$ & $\begin{array}{l}\text { Membre du comité directeur, député } \\
\text { (docteur) }\end{array}$ & Membre de la $1^{\text {re }}$ commission \\
\hline $\begin{array}{l}\text { Erich Ollenhauer } \\
\text { (1901-1963) }\end{array}$ & $\begin{array}{l}\text { Président, député (aucun diplôme } \\
\text { universitaire) }\end{array}$ & Membre de la $1^{\text {re }}$ commission \\
\hline $\begin{array}{l}\text { Fritz Sänger } \\
\text { (1901-1984) }\end{array}$ & $\begin{array}{l}\text { Journaliste (aucun diplôme universitaire, } \\
\text { formation d'instituteur) }\end{array}$ & Rapporteur de la $2^{\text {de }}$ commission \\
\hline $\begin{array}{l}\text { Otto Stammer } \\
(1900-1978)\end{array}$ & Professeur de sociologie (Berlin) & Membre de la $1^{\text {re }}$ commission \\
\hline $\begin{array}{l}\text { Gerhard Weisser } \\
\text { (1898-1989) }\end{array}$ & Professeur de politique sociale (Cologne) & Membre de la $1^{\text {re }}$ commission \\
\hline $\begin{array}{l}\text { Herbert Wehner } \\
(1906-1990)\end{array}$ & $\begin{array}{l}\text { Vice-président, député (aucun diplôme } \\
\text { universitaire) }\end{array}$ & Membre de la $1^{\text {re }}$ commission \\
\hline
\end{tabular}

